\title{
EFEITOS DO EXERCÍCIO RESISTIDO DE ALTA INTENSIDADE EM RATOS QUE RECEBERAM DEXAMETASONA
}

\author{
EFFECTS OF HIGH INTENSITY RESISTANCE TRAINING IN RATS \\ RECEIVING DEXAMETHASONE \\ EFECTOS DEL ENTRENAMIENTO RESISTIDO DE ALTA INTENSIDAD EN RATAS QUE \\ RECIBIERON DEXAMETASONA
}

Artigo Original

Original ARTICLE

Artículo Original

\begin{abstract}
Rejane Walessa Pequeno Rodrigues ${ }^{1,2,3}$

(Educadora Física e Nutricionista)

Odilon Abrahin 1,2,3

(Educador Físico)

Evitom Corrêa de Sousa ${ }^{3}$

(Educador Físico)

Anderson Carlos Marçal ${ }^{1,2}$

(Biólogo)

1. Universidade Federal de Sergipe, Departamento de Educação Física, Programa de Pós-Graduação em Educação Física, Sergipe, Brasil.

2. Universidade Federal de Sergipe, Departamento de Morfologia, Núcleo de Pesquisa em Sinalização Intracelular, Sergipe, Brasil.

3. Universidade do Estado do

Pará, Campus Educação Física,

Laboratório de Exercício Resistido

e Saúde, Pará, Brasil.
\end{abstract}

\section{Correspondência:}

Conjunto Cidade Nova 2, WE-18, 342. Ananindeua, PA, Brasil. 67130-460. rejanepequeno@r7.com

\section{RESUMO}

Introdução: A dexametasona administrada cronicamente promove alterações deletérias no metabolismo dos carboidratos. Objetivo: Avaliar os efeitos do exercício resistido de alta intensidade sobre a sensibilidade à insulina, tolerância à glicose e força muscular de ratos submetidos ao uso crônico de dexametasona. Métodos: Foram utilizados 40 ratos machos divididos randomicamente em quatro grupos: 1) Controle Sedentário (CS); 2) Controle Treinado (CT); 3) Dexametasona Sedentário (DS) e 4) Dexametasona Treinado (DT). O exercício resistido foi realizado em aparelho de agachamento composto por três séries, 10 repetições, com intensidade de $75 \%$ de 1 RM durante quatro semanas. Concomitantemente, os grupos DS e DT recebiam diariamente dexametasona intraperitoneal $(0,2 \mathrm{~g} / \mathrm{kg})$ e os grupos CS e CT recebiam somente solução salina $(0,9 \%)$. Ao final do protocolo foram realizados testes de tolerância à glicose, sensibilidade à insulina e teste de força máxima. Resultados: Nos grupos treinados (CT e DT) houve aumento da força muscular de 14,78\% e 36,87\% respectivamente, sem ganho significativo nos grupos sedentários. No teste de tolerância à glicose, os grupos treinados (CT e DT) apresentaram amplitudes atenuadas da glicose plasmática quando comparados aos grupos sedentários (CS e DS). No teste de sensibilidade à insulina, o grupo DT apresentou menor área sob a curva em relação ao grupo DS. Conclusão: $O$ exercício resistido de alta intensidade melhora a sensibilidade à insulina, tolerância à glicose e a força muscular em ratos que receberam a dexametasona.

Descritores: treinamento de resistência; resistência à insulina; hiperglicemia.

\section{ABSTRACT}

Introduction: Prolonged administration of dexamethasone promotes deleterious changes in the metabolism of carbohydrates. Objective: To evaluate the effects of high intensity resistance training on insulin sensibility, glucose tolerance, and muscle strength in rats submitted to chronic use of dexamethasone. Methods: Forty males rats were randomly divided into four groups: 1) Control Sedentary (CS); 2) Control Trained (CT); 3) Dexamethasone Sedentary (DS) and 4) Dexamethasone Trained (DT). Resistance training was conducted in squat apparatus composed of three series, 10 repetitions, with an intensity of $75 \%$ of 1 RM for four weeks. Concurrently, the DS and DT groups received daily intraperitoneal dexamethasone $(0.2 \mathrm{~g} / \mathrm{kg})$ and the CS and CT groups received only saline (0.9\%). At the end of the protocol, glucose tolerance, insulin sensibility and maximum force tests were conducted. Results: In the trained groups (CT e DT) was found an increased muscle strength of $14.78 \%$ and $36.87 \%$, respectively, with no significant gain in sedentary groups. In the glucose tolerance test, the trained groups (CT e DT) showed attenuated ranges of plasma glucose when compared to sedentary groups (CS and DS). In insulin sensibility test, the DT group showed lower area under the curve in relation to the DS group. Conclusion: The high intensity resistance exercise improved insulin sensibility, glucose tolerance and increased muscle strength in rats submitted to chronic use of dexamethasone.

Keywords: resistance training; insulin resistance; hyperglycemia.

\section{RESUMEN}

Introducción: A largo plazo, la dexametasona promueve cambios nocivos sobre el metabolismo de los hidratos de carbono. Objetivo: Evaluar los efectos del entrenamiento de resistencia de alta intensidad en la sensibilidad a la insulina, tolerancia a la glucosa y la fuerza muscular en ratas sometidas a la utilización crónica de dexametasona. Métodos: Se dividieron aleatoriamente 40 ratas macho en cuatro grupos: 1) Control Sedentario (CS); 2) Control Entrenado (CE) 3); Dexametasona Sedentario (DS); 4) Dexametasona Entrenado (DE). El entrenamiento de resistencia se llevó a cabo en un aparato de agachamiento compuesto por tres series, 10 repeticiones, con intensidad del 75\% de 1 RM durante cuatro semanas. Al mismo tiempo, los grupos de DS y DE recibieron dexametasona intraperitoneal diaria $(0,2 \mathrm{~g} / \mathrm{kg}$ ) y los grupos CS y CE recibieron sólo solución salina (0,9\%). Al final del protocolo se llevaron a cabo las pruebas de tolerancia a la glucosa, sensibilidad a la insulina y de fuerza máxima. Resultados: En los grupos entrenados (CE y DE) se aumentó la fuerza muscular del 14,78\% y 36,87\%, respectivamente, sin aumento significativo en los grupos sedentarios. En la prueba de tolerancia a la glucosa, 
los grupos entrenados (CEy DE) mostraron amplitudes atenuadas de la glucosa en plasma en comparación con los grupos sedentarios (CS y SD). En la prueba de sensibilidad a la insulina, el grupo DE mostró disminución del área bajo la curva en relación con el grupo DS. Conclusión: El entrenamiento de resistencia de alta intensidad promovió mejora en la sensibilidad a la insulina, tolerancia a la glucosa y el aumento de la fuerza muscular en ratas sometidas al uso crónico de dexametasona.

Descriptores: entrenamiento de resistencia; resistencia a la insulina; hiperg/ucemia.

\section{INTRODUÇÃO}

A alta incidência de doenças inflamatórias ou que contribuem para o desenvolvimento de um quadro inflamatório, como as reumáticas e até alguns tipos de câncer podem estar relacionadas com a prescrição e uso crônico de diversos medicamentos, como os glicocorticoides (dexametasona), utilizado pelo seu potencial benefício anti-inflamatório'.

Todavia, a dexametasona utilizada em grande quantidade está relacionada à síndrome de Cushing iatrogênica, caracterizada pela "face de lua", surgimento de estrias, ganho de peso com acúmulo de gordura central. Além destes efeitos, a utilização indiscriminada de glicocorticoides promove alterações negativas no metabolismo da glicose ${ }^{2,3}$, miopatia e fraqueza muscular ${ }^{4-6}$, hipertensão arterial ${ }^{7,8}$, osteoporose $e^{9,10}$ e maior suscetibilidade a infecções oportunistas ${ }^{11}$.

Nesse sentido, buscam-se métodos alternativos que possam reduzir/atenuar os efeitos adversos decorrentes do uso crônico da dexametasona. Dentre estas, os exercícios físicos apresentam-se como um possível recurso não farmacológico, contrapondo os efeitos deletérios provocados pelo uso contínuo deste medicamento ${ }^{12-14}$.

O exercício resistido (ER) se destaca por melhorar a composição corporal, aumentar a síntese proteica, força muscular ${ }^{15}$, alterar positivamente o perfil lipídico e promover ajustes pressóricos ${ }^{16}, \mathrm{e}$, sobretudo, aumentar a sensibilidade à insulina e tolerância à glicose ${ }^{17,18}$.

Desta forma, a principal hipótese deste estudo considerou que o exercício resistido pode atenuar/reduzir os efeitos adversos causados pelo uso continuado de dexametasona. Logo, o objetivo deste estudo foi avaliar os efeitos do exercício resistido de alta intensidade sobre a sensibilidade à insulina, tolerância à glicose e força muscular em ratos submetidos ao uso crônico de dexametasona por 30 dias consecutivos.

\section{MÉTODOS}

Foram utilizados ratos Wistar machos (Rattus Norvegicus albinus Wistar), com idade de 7 a 8 semanas, pesando entre $250 \mathrm{~g}$ e $300 \mathrm{~g}$. Os animais foram acomodados em número de 5 por caixa retangular grande, mantidos sob condições de temperatura controlada de $21^{\circ}$ a $24^{\circ} \mathrm{C}$ e ciclo claro-escuro de 12 horas, tendo livre acesso à água e alimentação (Purina ${ }^{\circledR}$, Presence, Brasil).

Os procedimentos descritos neste estudo foram previamente aprovados pelo comitê de ética em pesquisa animal da universidade local (CEPA/UFS) sob o número de protocolo 09/2012.

Os animais foram distribuídos aleatoriamente em quatro grupos:

- Controle Sedentário (CS): ratos sedentários e que receberam solução salina $(0,9 \%)$ intraperitonialmente;

- Controle Treinado (CT): ratos que foram submetidos somente ao protocolo de ER e receberam solução salina (0,9\%) intraperitonialmente;

- Dexametasona Sedentário (DS): ratos sedentários que foram submetidos somente à administração de dexametasona;

- Dexametasona Treinado (DT): ratos que foram submetidos ao protocolo de ER e à administração de dexametasona.

O ER foi realizado em aparelho de agachamento segundo modelo de Tamaki et al. ${ }^{19}$. Todos os animais foram familiarizados com duas sessões de treino (três vezes a massa corporal, 10 repetições, duas séries, um minuto de intervalo) com intervalo de 48 horas entre as sessões. Os grupos CT e DT foram submetidos ao treinamento três vezes por semana executando três séries de dez repetições, com intervalos de repouso de 60 segundos e intensidade de 75\% da carga estabelecida através do teste de uma repetição máxima (1RM).

O protocolo de treinamento teve duração de quatro semanas. Os animais executaram as séries através da aplicação de estímulos elétricos (20 V, 0.3s de duração, 3s de intervalo) por eletrodos (ValuTrode, Modelo (F3200, Axelgaard, Fallbrook, CA, EUA) fixados na cauda e conectados a um eletroestimulador (BIOSET, Physiotonus Four, Modelo 3050, Rio Claro, SP, Brasil).

Os grupos CS e DS foram fixados no aparelho nos mesmos horários de treinamento dos grupos exercitados e apenas submetidos aos estímulos elétricos (três séries, dez repetições, com intervalos de repouso de 60 segundos) sem qualquer realização do movimento específico do exercício.

Após a familiarização, todos os grupos realizaram o teste de uma repetição máxima (1RM) e após 48 horas foi realizado o reteste de (1RM). Para reajustar a carga de treino e manter a intensidade proposta foram realizados novos testes de 1 RM a cada duas semanas.

Foi administrado dexametasona durante 30 dias, uma vez ao dia (0,2 mg/kg/dia, Decadron ${ }^{\circledR}$, Prodome, Brasil) intraperitonialmente sempre no mesmo horário (13 horas) nos animais DS e DT. Segundo Severino e colaboradores essa dose administrada é capaz de induzir alterações metabólicas ${ }^{20}$. Nos animais CS e CT, foram injetados intraperitonialmente solução salina (0,9\%) no mesmo horário (às 13 horas).

A massa corporal foi monitorada 3 vezes por semana no mesmo horário em todos os grupos experimentais e o protocolo de treinamento foi realizado concomitante à administração da dexametasona.

Ao término dos protocolos experimentais, os animais de cada grupo foram submetidos ao ivGTT após restrição alimentar de 12 horas e foram anestesiados com tiopental ( $40 \mathrm{mg} / \mathrm{Kg}$ de peso de animal). Ao se confirmar o efeito do anestésico pela observação da não retirada da cauda após estímulo de pressão, foi coletado por punção caudal, a amostra correspondente ao tempo zero do teste. Sendo injetado a solução de glicose ( $2 \mathrm{~g} / \mathrm{kg}$ de peso corporal, via veia peniana) diluída em solução salina (0,9\%).

Coletaram-se amostras de sangue por punção caudal nos tempos 5, 15, 30, 60 e 90 minutos após injeção de glicose. A glicemia foi determinada através do glicosímetro (Accu-chek Active - Roche). As áreas sob curvas de glicose durante o ivGTT foram calculadas pelo método trapezoidal21 no software GraphPrism versão 5.

Para avaliar a sensibilidade à insulina in vivo, os animais foram anestesiados com tiopental (40 mg/Kg de peso de animal). O tiopental foi utilizado por não apresentar influencia na via de sinalização da insulina ${ }^{22}$. Ao se confirmar o efeito do anestésico pela observação da não retirada da cauda após estímulo de pressão, foi administrada insulina na dose de $5,4 \mathrm{mmol}^{-1} / \mathrm{kg}$ de peso corpóreo na veia peniana e foi analisada a concentração de glicose plasmática em diferentes tempos de coleta de sangue (0 - tempo basal, 4, 8, 12, 16, 18 e 30 minutos) após a administração da insulina23,24. 


\section{Análise dos Dados}

A avaliação estatística foi realizada pelo teste $t$-Student para 2 amostras não pareadas $(p<0,05)$; também foi utilizado teste de uma via (One way - ANOVA) e de duas vias (Two way - ANOVA), para mais de 4 variáveis, utilizando para ambos o pós-teste de Bonferroni $(p<0,05)$.

\section{RESULTADOS}

Para avaliarmos se o protocolo de exercício resistido utilizado promoveu ganho de força e também para ajustar a intensidade de treinamento, os animais foram submetidos ao teste de força máxima (Figura 1). Não observamos diferenças significativas no teste de 1RM nos grupos sedentários (CS e DS) entre a primeira e quarta semana do protocolo (Figura 1A). Em relação aos grupos treinados (CT e DT) houve um aumento na força, em 14,78\% e 36,87\%, respectivamente, após período de intervenção (Figura 1B).

O grupo controle sedentário (CS) apresentou ganho de peso ao longo do tempo investigado, este efeito foi semelhante no grupo controle treinado (CT). Os animais tratados com dexametasona tanto no grupo sedentário (DS) quanto no grupo treinado (DT) apresentaram redução de peso corporal (Figura 2).

Durante o teste de tolerância à glicose (ivGTT) (Figura 3), os animais pertencentes aos grupos controle sedentário (CS) e controle treinados (CT) após a administração intravenosa de glicose, demonstraram aumento da concentração plasmática de glicose. Todavia, a amplitude da glicose plasmática do grupo controle treinado (CT), foi atenuado quando comparado ao grupo controle sedentário (CS) (Figura 3A), esta redução foi de 12,99\% segundo área sob a curva (Figura 3B).

Os animais pertencentes aos grupos dexametasona (DS e DT)

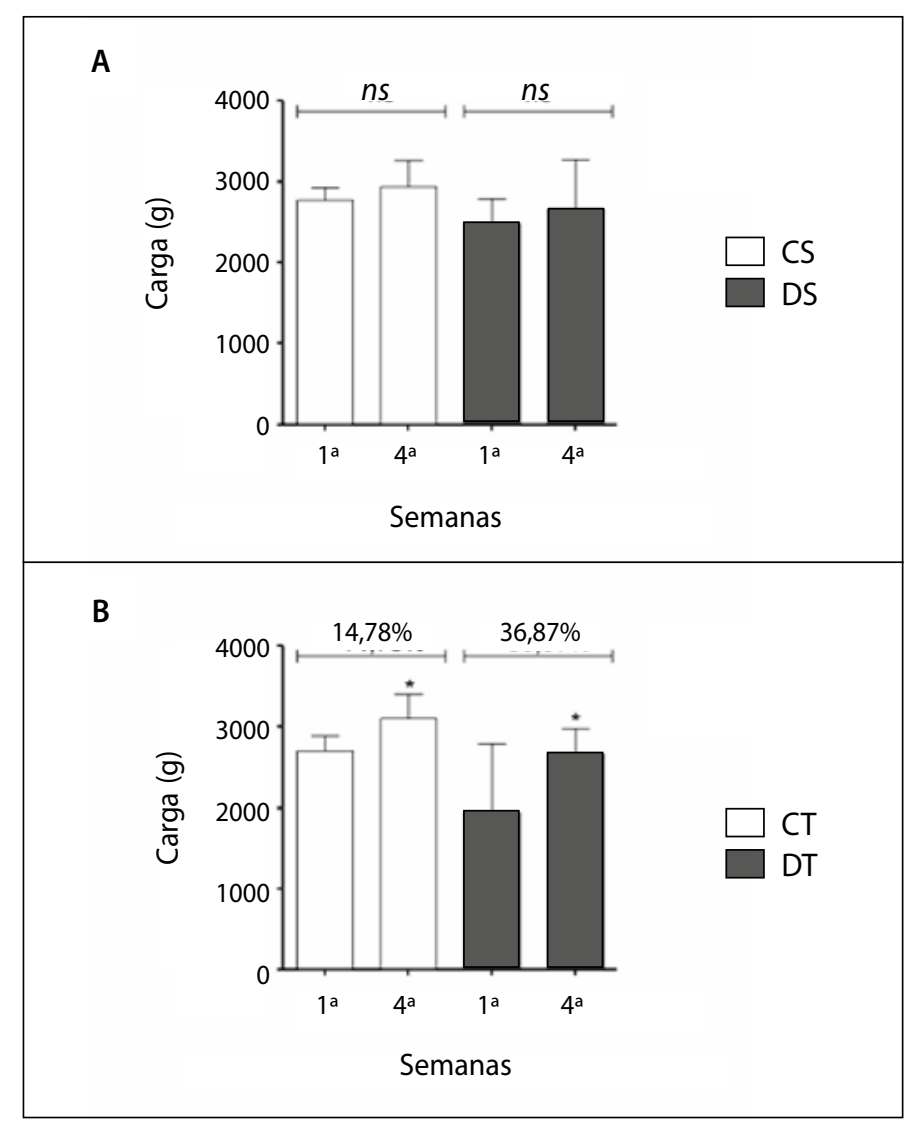

Figura 1. Teste de Força Máxima (1RM) antes e depois do protocolo. Os dados representam a média \pm desvio padrão da média. A Figura $1 \mathrm{~A}$, ilustra os resultados dos grupos controle sedentário (CS; $n=10$ ) dexametasona sedentário (DS; $n=10)$. A Figura $1 \mathrm{~B}$, ilustra os resultados dos grupos controle treinado $(C T ; n=10)$ e dexametasona treinado ( $D T ; n=10)$. Utilizou-se o teste t de Student para análise entre os grupos. ns $=$ sem diferença estatística; ${ }^{*} p<0,05$.

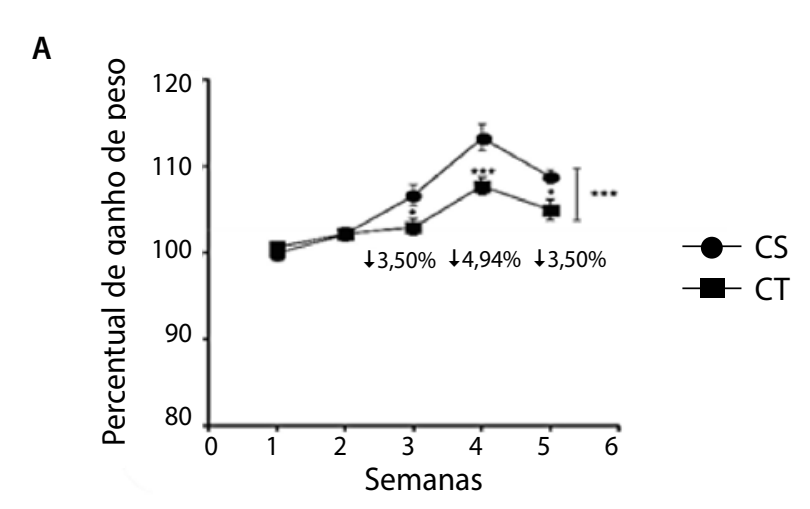

B

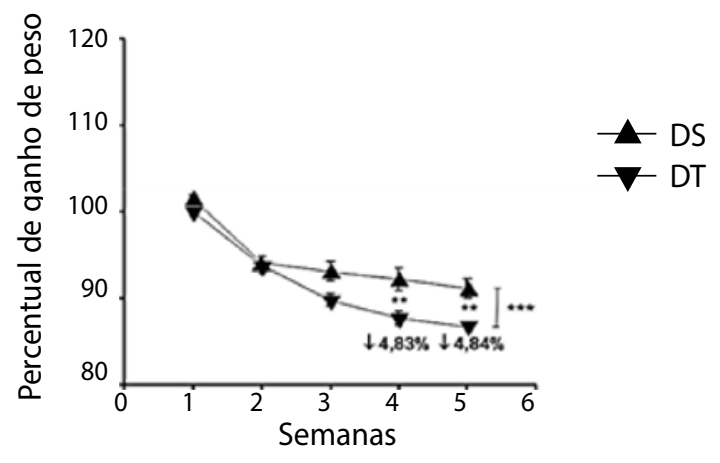

Figura 2. Avaliação do percentual do peso corporal total dos grupos (A) Grupo Controle Sedentário (CS) e Grupo Controle Treinado (CT). (B) Grupo Dexametasona Sedentário (DS) e Grupo Dexametasona Treinado (DT). $(n=10)$ (Teste de duas vias com pós-teste de Bonferroni); ${ }^{*} p<0,05 ;{ }^{* *} p<0,01 ;{ }^{* * *} p<0,001$.

após a administração endovenosa de glicose, também demonstraram aumento da concentração plasmática de glicose. Todavia, a amplitude da glicose plasmática do grupo dexametasona treinado (DT) foi reduzido quando comparado ao grupo dexametasona sedentário (DS) (Figura 3C), esta redução foi de 22,33\% segundo área sob a curva (Figura 3D).

Com relação ao teste de sensibilidade à insulina (ivITT) (Figura 4), como esperado, os grupos controles (CS e CT) apresentaram declínio progressivo na concentração da glicose plasmática após a administração endovenosa de insulina. Todavia, não apresentaram diferença significativa (Figura 4A). Os grupos tratados com dexametasona (DS e DT), também apresentam declínio da concentração plasmática de glicose após a administração de insulina (Figura 4C). Porém no grupo dexametasona treinado (DT) a área sob a curva foi 23,81\% menor em relação ao grupo dexametasona sedentário (DS) (Figura 4D).

\section{DISCUSSÃO}

O principal resultado desde estudo evidenciou que o exercício resistido de alta intensidade melhorou a sensibilidade à insulina e atenuou a hiperglicemia em ratos submetidos ao uso de dexametasona. É consenso na literatura científica os efeitos adversos ocasionados pelo uso crônico da dexametasona principalmente, no metabolismo do carboidrato $2,3,12,13$.

Pinheiro et al ${ }^{13}$, evidenciaram intolerância à glicose e resistência à insulina em animais que receberam dexametasona por 30 dias consecutivos $(5 \mathrm{mg} / \mathrm{kg} / \mathrm{dia})$. A resistência à ação da insulina pode estar diretamente associada a redução de 30-50\% da captação de glicose estimulada por este hormônio ${ }^{25}$. Além disto, a síntese de glicogênio estimulada pela insulina também é prejudicada em 70\% após o tratamento com glicocorticoide ${ }^{12}$. 


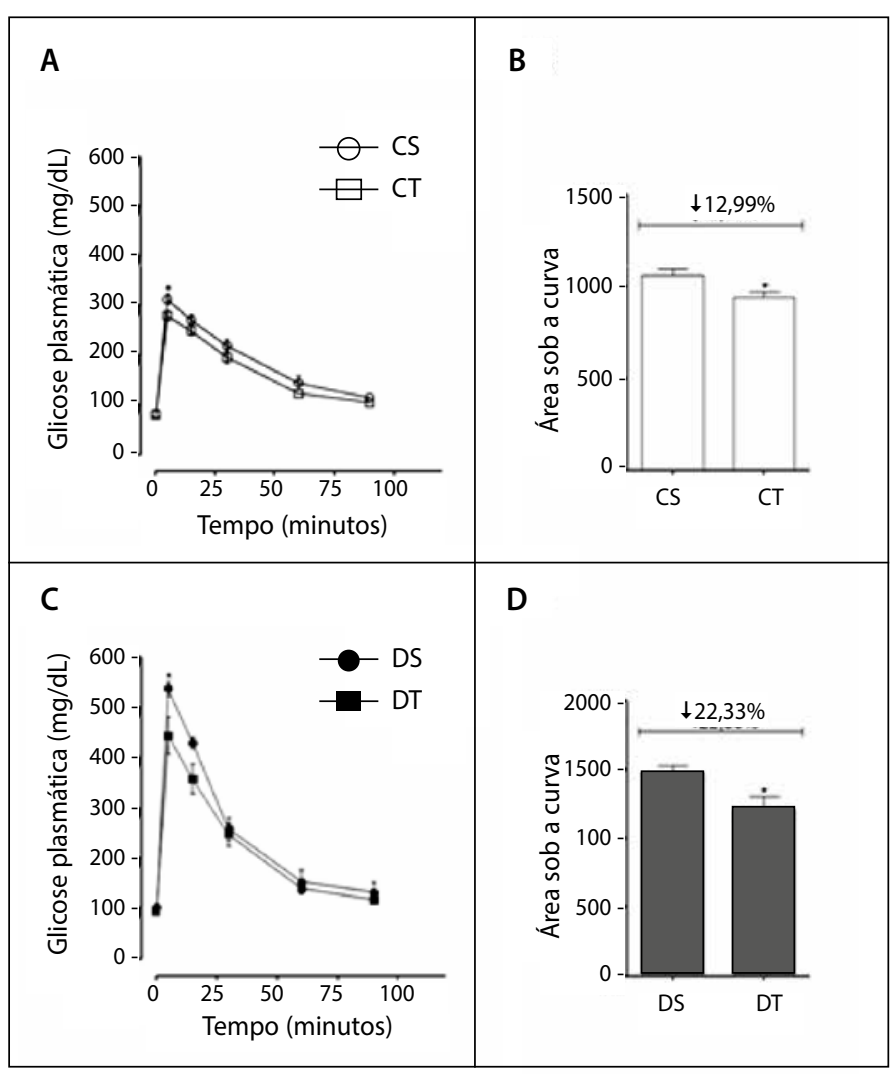

Figura 3. Teste de tolerância a glicose (ivGTT). (A) Concentração da glicose plasmática dos grupos controle sedentário (CS) e controle treinado (CT) após a sobrecarga de glicose, os resultados foram representados na forma de um gráfico de linha. (B) Àrea sob a curva dos grupos CS e CT. (C) Para os grupos dexametasona sedentários (DS) e dexametasona treinados (DT), o gráfico de linha e a área sob a curva estão ilustrados nas figuras 3C e 3D, respectivamente. Foram utilizados o teste t-Student para análise entre duas variáveis e teste de duas vias (Two Way) com pós-teste de Bonferroni para múltiplas variáveis). ns $=$ sem diferença estatística; ${ }^{*} p<0,05$.

Estes efeitos sobre o metabolismo dos carboidratos podem ser evidenciados a partir de $2 \mu \mathrm{g} /$ dia de dexametasona por via a via subcutânea ${ }^{20}$. A partir destes resultados, neste estudo foi administrado 0,2mg/kg/dia.

Para avaliação da tolerância à glicose e sensibilidade à insulina utilizamos dois testes funcionais conhecidos mundialmente baseados no guideline "Avaliação da homeostase em modelos de roedores" ${ }^{26}$. Nossos resultados demonstraram que o uso crônico de dexametasona alterou a resposta glicêmica durante os diferentes protocolos após a sobrecarga de glicose durante o teste de tolerância a glicose (ivGTT), corroborado por outros autores ${ }^{10,12,13}$.

Os grupos de animais submetidos ao exercício resistido, tanto o grupo controle treinado (CT) quanto o grupo dexametasona treinado (DT), após a sobrecarga com glicose, apresentaram redução da amplitude da concentração de glicose plasmática quando comparados com os grupos controle sedentário (CS) e dexametasona sedentário (DS), respectivamente. Estes resultados sugerem, possivelmente, aumento da captação da glicose em ambos os grupos treinados (CT e DT).

De acordo com Winett e Carpinelli27 o exercício resistido é capaz de promover ajustes benéficos sobre a concentração de glicose e homeostase da insulina, uma vez que, este efeito está diretamente relacionado com o aumento da área da secção transversa muscular e massa corporal magra promovido pela prática de exercício resistido.

Além destas alterações macroscópicas, estes mesmos autores ${ }^{27}$ evidenciaram aumento na expressão gênica do GLUT-4, do índice de translocação desta molécula à superfície celular, bem como aumento do conteúdo/atividade da enzima glicogênio síntase. Estas proteínas estão envolvidas diretamente na captação de glicose e síntese de glicogênio, respectivamente ${ }^{17,18,28-31}$.

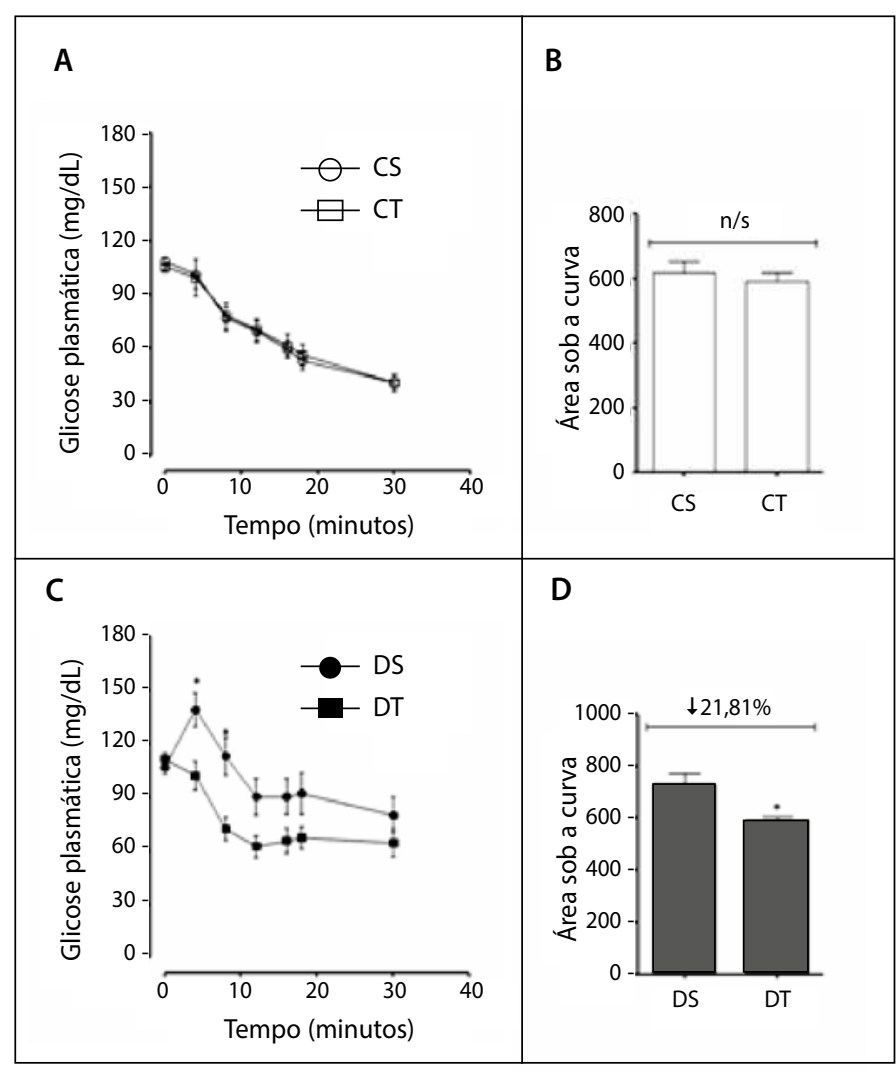

Figura 4. Teste de sensibilidade a insulina (ivITT). O decaimento da glicemia plasmática do grupo controle sedentário (CS) e controle treinado (CT) após a administração endovenosa de insulina durante o ivITT, os dados foram representados na forma de um gráfico de linha (Figura 4A) e na área sob a curva (figura 4B). Para os grupos com dexametasona sedentário e treinado (DS e DT), o gráfico de linha e a área sob a curva estão ilustrados nas figuras 4C e 4D, respectivamente. Foram utilizados o teste t-Student para análise entre duas variáveis e teste de duas vias (Two Way) com pós-teste de Bonferroni para múltiplas variáveis). ns = sem diferença estatística; ${ }^{*} p<0,05$.

A prática do exercício resistido pode contribuir para a melhora da glicemia através de três possíveis mecanismos: contração muscular, produção de adenosina monofosfato (AMP) e ativação da proteína quinase ativada pelo AMP (AMPK) $28,29,30$. Holmes e Dohm ${ }^{31}$ sugerem que a melhora da captação de glicose pode ser explicada pelo aumento da expressão proteica e translocação de GLUT-4 do retículo endoplasmático em direção à membrana plasmática. Esta proteína é modulada durante o exercício pela $\mathrm{Ca}^{++} / \mathrm{Calmodulina} \mathrm{quinase} \mathrm{(CaMK)}{ }^{31}$.

A concentração intracelular de AMPK e CaMK, durante o exercício resistido encontram-se aumentada e ambas estimulam a fosforilação do substrato (AS160) $)^{31,32}$, estes eventos culminam com a translocação de GLUT-4 para a membrana celular, desencadeando assim, a captação de glicose.

Para que estes benefícios sejam significativos, é necessário que a prática de exercício resistido seja ajustada, respeitando a individualidade biológica e as variáveis do treinamento, além disso, a intensidade utilizada neste estudo foi $75 \%$, considerado como treinamento de alta intensidade ${ }^{27,32}$.

Além dos benefícios positivos discutidos anteriormente, outro parâmetro de análise foi o de força muscular, avaliado através do teste de 1RM, como indicador de eficácia do treinamento, demostrando que os grupos treinados (CT e DT) ganharam mais força quando comparados aos grupos sedentários (CS e DS).

O grupo dexametasona treinado DT obteve ganho de força mesmo sob efeito do medicamento, demonstrando o benefício do treinamento. De acordo com a literatura ${ }^{33,34}$, sujeitos com baixos níveis de aptidão física possuem a tendência em apresentar maior magnitude de adaptações morfofuncionais, essa observação é denominada como princípio da treinabilidade ${ }^{34}$. 
O modelo de treinamento utilizado foi o de agachamento, elaborado por TAMAKI e colaboradores ${ }^{19}$ o qual tem sido aplicado em diversos trabalhos para mimetizar respostas semelhantes às obtidas em humanos adeptos ao exercício resistido ${ }^{35-37}$.

Um dos primeiros efeitos adversos causados pela dexametasona foi a diminuição progressiva de massa corporal total ${ }^{10,12-13}$. Neste estudo, os grupos controles (CS e CT) apresentaram ganho progressivo do percentual de massa corporal durante as 4 semanas do protocolo. Nos grupos DS e DT, houve perda no percentual da massa corporal durante todo o protocolo.

O presente estudo foi pioneiro em utilizar exercício resistido de alta intensidade em animais administrados com dexametasona, um estudo utilizando exercício resistido de baixa intensidade demonstrou atenuação na atrofia do músculo flexor longo do halux ${ }^{38}$ porém, também identificou perda de massa corporal nos animais submetidos ao dexametasona. Estes dados corroboram com Barel et al. ${ }^{12}$ e Pinheiro et al. ${ }^{13}$ porém, estes autores utilizaram exercício aeróbio.

A prescrição e o uso da dexametasona, aparentemente, podem ser controversos, por apresentar grande utilidade por seus efeitos anti-inflamatórios e imunossupressores. Porém, quando seu uso for prolongado ou administrado em doses suprafisiológicas pode ser capaz de promover efeitos deletérios.

Desta forma, como medidas profiláticas ou que objetivam atenuar esses efeitos adversos, o exercício resistido parece ser uma alternativa importante a ser utilizada para atenuar os quadros de hiperglicemia e de resistência à ação da insulina em pacientes usuários de dexametasona. E, contribuir na diminuição do risco de desenvolver alterações metabólicas que possam resultar no diabetes e outras doenças.

\section{CONCLUSÕES}

Os resultados do nosso estudo evidenciam que o exercício resistido melhora a sensibilidade à insulina, tolerância à glicose e a força muscular em ratos que receberam cronicamente a dexametasona.

Todos os autores declararam não haver qualquer potencial conflito de interesses referente a este artigo.

CONTRIBUIÇÕES DOS AUTORES: Cada autor contribuiu individual e significativamente para o desenvolvimento do manuscrito. RWPR (0000-0003-3365-5444)* , OSCA (0000-0003-3750-2517)* e ACM (0000-0001-7869-2922)* foram os principais contribuintes na redação do manuscrito e reuniram os dados clínicos a partir da execução dos experimentos. ACM avaliou os dados da análise estatística. RWPR, OSCA, ACM e ECS (0000-0002-4002-239X)* realizaram a pesquisa bibliográfica, a revisão do manuscrito e contribuíram com o conceito intelectual do estudo. *Número ORCID (Open Researcher and Contributor ID).

\section{REFERÊNCIAS}

1. Direcção-Geral da Saúde. Divisão de Doenças Genéticas, Crônicas e Geriátricas. Programa nacional contra as doenças reumáticas. Lisboa: DGS; 2005. p. 92.

2. Liu XX, Zhu XM, Miao Q, Ye HY, Zhang ZY, Li YM. Hyperglycemia induced by glucocorticoids in nondiabetic patients: a meta-analysis. Ann Nutr Metab. 2014;65(4):324-32.

3. Perez A, Jansen-Chaparro S, Saigi I, Bernal-Lopez MR, Miñambres I, Gomez-Huelgas R. Glucocorticoid-induced hyperglycemia. J Diabetes. 2014;6(1):9-20.

4. Qin J, Du R, Yang YQ, Zhang HQ, Li Q, Liu L, et al. Dexamethasone-induced skeletal muscle atrophy was associated with upregulation of myostatin promoter activity. Res Vet Sci. 2013;94(1):84-9

5. Ma K, Mallidis C, Bhasin S, Mahabadi V, Artaza J, Gonzalez-Cadavid N, et al. Glucocorticoid-induced skeletal muscle atrophy is associated with upregulation of myostatin gene expression. Am J Physiol Endocrinol Metab. 2003;285(2):E363-71.

6. Schakman O, Kalista S, Barbé C, Loumaye A, Thissen JP. Glucocorticoid-induced skeletal muscle atrophy. Int J Biochem Cell Biol. 2013;45(10):2163-72.

7. Goodwin JE, Geller DS. Glucocorticoid-induced hypertension. Pediatr Nephrol. 2012;27(7):1059-66.

8. Saruta T. Mechanism of glucocorticoid-induced hypertension. Hypertens Res. 1996;19(1):1-8.

9. Warriner AH, Saag KG. Glucocorticoid-related bone changes from endogenous or exogenous glucocorticoids. Curr Opin Endocrinol Diabetes Obes. 2013;20(6):510-6.

10. De Nijs RN. Glucocorticoid-induced osteoporosis: a review on pathophysiology and treatment options. Minerva Med. 2008;99(1):23-43.

11. Faiçal S, Uehara MH. Efeitos sistêmicos e síndrome de retirada em tomadores crônicos de corticosteróides. Rev Assoc Med Bras. 1998:44(1):69-74.

12. Barel M, Perez OA, Giozzet VA, Rafacho A, Bosqueiro JR, do Amaral SL. Exercise training prevents hyperinsulinemia, muscular glycogen loss and muscle atrophy induced by dexamethasone treatment. Eur J Appl Physiol. 2010;108(5):999-1007.

13. Pinheiro CH, Sousa Filho WM, Oliveira Neto J, Marinho M J, Motta Neto R, Smith MM, et al. Exercise prevents cardiometabolic alterations induced by chronic use of glucocorticoids. Arq Bras Cardiol. 2009;93(4):400-8, 392-400.

14. Bouchard C, Antunes-Correa LM, Ashley EA, Franklin N, Hwang PM, Mattsson CM, et al. Personalized preventive medicine: genetics and the response to regular exercise in preventive interventions. Prog Cardiovasc Dis. 2015:57(4):337-46.

15. Ciolac EG, Greve JM. Muscle strength and exercise intensity adaptation to resistance training in older women with knee osteoarthritis and total knee arthroplasty. Clinics (Sao Paulo). 2011;66(12):2079-84.

16. Westcott WL. Resistance training is medicine: effects of strength training on health. Curr Sports Med Rep. 2012:11(4):209-16

17. Eves ND, Plotnikoff RC. Resistance training and type 2 diabetes: Considerations for implementation at the population level. Diabetes Care. 2006;29(8):1933-41.

18. Colberg SR, Sigal RJ, Fernhall B, Regensteiner JG, Blissmer BJ, Rubin RR, et al. Exercise and type 2 diabetes: the American College of Sports Medicine and the American Diabetes Association: joint position statement. Diabetes Care. 2010;33(12):e147-67.

19. Tamaki T, Uchiyama S, Nakano S. A weight-lifting exercise model for inducing hypertrophy in the hindlimb muscles of rats. Med Sci Sports Exerc. 1992;24(8):881-6.

20. Severino C, Brizzi P, Solinas A, Secchi G, Maioli M, Tonolo G. Low-dose dexamethasone in the rat: a model to study insulin resistance. Am J Physiol Endocrinol Metab. 2002;283(2):E367-73.

21. Matthews JN, Altman DG, Campbell MJ, Royston P. Analysis of serial measurements in medical research. BMJ. 1990;300(6719):230-5.
22. Cardoso AR, Carvalho CR, Velloso LA, Brenelli SL, Saad MJ, Carvalheira JB. Effect of thiopental, pentobarbital and diethyl ether on early steps of insulin action in liver and muscle of the intact rat. Life Sci. 2005;76(20):2287-97.

23. Bonora E, Moghetti P, Zancanaro C, Cigolini M, Querena M, Cacciatori V, et al. Estimates of in vivo insulin action in man: comparison of insulin tolerance tests with euglycemic and hyperglycemic glucose clamp studies. J Clin Endocrinol Metab. 1989:68(2):374-8.

24. Marçal AC, Camporez JP, Lima-Salgado TM, Cintra DE, Akamine EH, Ribeiro LM, et al. Changes in food intake, metabolic parameters and insulin resistance are induced by an isoenergetic, medium-chain fatty acid diet and are associated with modifications in insulin signalling in isolated rat pancreatic islets. Br J Nutr. 2013;109(12):2154-65.

25. Nicastro H, Gualano B, de Moraes WM, de Salles Painelli V, da Luz CR, dos Santos Costa A, et al. Effects of creatine supplementation on muscle wasting and glucose homeostasis in rats treated with dexamethasone. Amino Acids. 2012;42(5):1695-701.

26. Bowe JE, Franklin ZJ, Hauge-Evans AC, King AJ, Persaud SJ, Jones PM. Metabolic phenotyping guidelines: assessing glucose homeostasis in rodent models. J Endocrinol. 2014;222(3):G13-25.

27. Winett RA, Carpinelli RN. Potential health-related benefits of resistance training. Prev Med. 2001;33(5):503-13.

28. Egan B, Zierath JR. Exercise metabolism and the molecular regulation of skeletal muscle adaptation. Cell Metab. 2013:17(2):162-84

29. Bweir S, Al-Jarrah M, Almalty AM, Maayah M, Smirnova IV, Novikova L, et al. Resistance exercise training lowers $\mathrm{HbA} 1 \mathrm{c}$ more than aerobic training in adults with type 2 diabetes. Diabetol Metab Syndr. 2009;1:27.

30. Hawley JA, Lessard SJ. Exercise training-induced improvements in insulin action. Acta Physiol. 2008;192(1):127-35.

31. Holmes B, Dohm GL. Regulation of GLUT4 gene expression during exercise. Med Sci Sports Exerc 2004;36(7):1202-6.

32. Adams K, Cafarelli E, Gary A, Dooly C, Matthew S, Fleck SJ, et al. Progression models in resistance training for healthy adults. Med Sci Sports Exerc. 2009:41(3):687-708.

33. Garber CE, Blissmer B, Deschenes MR, Franklin BA, Lamonte MJ, Lee IM, et al. American College of Sports Medicine position stand. Quantity and quality of exercise for developing and maintaining cardiorespiratory, musculoskeletal, and neuromotor fitness in apparently healthy adults: guidance fo prescribing exercise. Med Sci Sports Exerc. 2011;43(7):1334-59

34. Prestes J, Foschini D, Marchetti P, Charro MA. Prescrição e periodização do treinamento de força em academias. São Paulo: Manole; 2010.

35. Araujo AJ, Santos AC, Souza Kdos S, Aires MB, Santana-Filho VJ, Fioretto ET, et al. Resistance training controls arterial blood pressure in rats with L-NAME- induced hypertension. Arq Bras Cardiol. 2013;100(4):339-46.

36. Silva TLTB, Mota MM, Fontes MT, Soares APS, Barreto AS, Marçal AC, et al. Suplementação com L-arginina associada ao exercício resistido melhora a força muscular e impede o aumento da glicemia de ratos diabéticos. Rev Cienc Med Biol. 2010;12(1):89-93.

37. Stefani GP, Nunes RB, Dornelles AZ, Alves JP, Piva MO, Domenico MD, et al. Effects of creatine supplementation associated with resistance training on oxidative stress in different tissues of rats. J Int Soc Sports Nutr. 2014;11(1):11.

38. Macedo AG, Krug AL, Herrera NA, Zago AS, Rush JW, Amaral SL. Low-intensity resistance training attenuates dexamethasone-induced atrophy in the flexor hallucis longus muscle. J Steroid Biochem Mol Biol. 2014;143:357-64. 